\title{
Questes
}

Revue pluridisciplinaire d'études médiévales

\section{Variations sur une liste d'amants malheureux dans les Romans de Troie : vers un timide reflet de la thématique des Hommes illustres?}

\section{Anne Rochebouet}

\section{(2) OpenEdition}

\section{Journals}

Édition électronique

URL : http://journals.openedition.org/questes/509

DOI : 10.4000 /questes.509

ISSN : 2109-9472

Éditeur

Les Amis de Questes

\section{Édition imprimée}

Date de publication : 15 octobre 2009

Pagination : 89-98

ISSN : 2102-7188

\section{Référence électronique}

Anne Rochebouet, « Variations sur une liste d'amants malheureux dans les Romans de Troie : vers un timide reflet de la thématique des Hommes illustres ? », Questes [En ligne], 17 | 2009, mis en ligne le 01 janvier 2014, consulté le 21 avril 2019. URL : http://journals.openedition.org/questes/509 ; DOI :

10.4000 /questes.509 


\title{
Variations sur une liste d'amants malheureux dans les Romans de Troie : vers un timide reflet de la thématique des Hommes illustres?
}

\author{
Anne RochebOUET
}

Le recours à la liste, notamment de noms propres, est une pratique d'écriture médiévale bien connue ${ }^{1}$. Il s'agit aussi de la première forme qui sert d'expression, dans le domaine linguistique français, à la thématique des Hommes illustres : avant les recueils de biographies qui apparaîtront en France à la fin $\mathrm{du} X \mathrm{XV}^{\mathrm{e}}$ siècle et fleuriront au $\mathrm{XVI}^{\mathrm{e}}$ siècle ${ }^{2}$, les énumérations d'hommes célèbres se lisent parfois comme de véritables mises en série de personnages réels, ou en tout cas considérés comme tels, réunis à des fins d'exemplarité, et dans la lignée desquels s'inscrit, de façon plus ou moins explicite, un personnage, l'auteur ou encore le commanditaire du texte. Toutes les listes, loin de là, ne sont pas pour autant des illustrations de cette thématique : l'énumération d'exemples est en premier lieu un moyen pour les médiévaux d'exposer les autorités auxquelles ils ont recours et sur lesquelles ils appuient leurs propos.

Quelle que soit l'utilisation qui est faite de ces listes, on sait qu'à cette forme figée correspond un contenu tout aussi topique : s'y énumèrent un nombre limité de réalités. Parmi celles-ci figurent les listes d'amants, et notamment d'amants malheureux (ou d'hommes trompés par une femme), qui sont nombreuses dans les textes médiévaux. L'exemple littéraire le plus connu se lit dans La Mort le roi $\mathrm{Artu}^{3}$ où Bohort convoque, pour illustrer le rôle néfaste de l'amour, le fils de David, Salomon, Samson, Hector et Achille, et enfin Tristan.

La matière troyenne est un lieu d'expression tout trouvé pour cette

\footnotetext{
${ }^{1}$ Madeleine JEAY y a d'ailleurs consacré récemment une étude : Le Commerce des mots : l'usage des listes dans la littérature médiévale (XII'-XV siècles), Genève, Droz, 2006.

${ }^{2}$ Cf. Patricia EICHEL-LoJKInE, Le Siècle des grands hommes. Les recueils de vies d'hommes illustres avec portraits du XVI siècle, Leuven, Peeters, 2001.

3 Édition Jean FrapPIER, Droz, 1996 (3 ${ }^{\mathrm{e}}$ éd.), § 59, 1. 31-60.
} 
séquence topique énumérative prouvant la folie amoureuse. On voudrait ici s'arrêter sur une incarnation de cette pratique et sur l'évolution de son utilisation au sein de deux Romans de Troie en prose, Prose 1 et Prose $5^{4}$, textes d'époques différentes, mais liés entre eux par des rapports de copie ou de réécriture. Cette liste de sept amants malheureux apparait, à une seule reprise, pour illustrer la folie provoquée par l'amour, à propos du comportement d'Achille ${ }^{5}$.

Afin de mieux mettre en évidence la place au sein de la matière troyenne de ces deux cuvres, on dressera un rapide panorama de la diffusion médiévale de l'histoire troyenne, avant de s'arrêter sur les différentes formes que prend cette liste d'amants et de s'interroger sur leur lien avec la thématique des Hommes illustres.

\section{La diffusion de la matière troyenne au Moyen Âge dans la lignée du} Roman de Troie

L'histoire de la guerre de Troie est au Moyen Âge un véritable bestseller : Bernard Guenée ${ }^{6}$ rappelle en effet qu'elle a connu une diffusion que ne dépasse que celle de l'Histoire Sainte. Contrairement à aujourd'hui, ce n'est pas Homère qui sert de source aux hommes médiévaux, mais des textes postérieurs apocryphes : Darès le phrygien du côté troyen et Dictys de Crète, du côté grec ${ }^{7}$.

${ }^{4}$ Prose 1 a été partiellement éditée par Léopold CONSTANS et Edmond FARAL, Paris, Champion, 1922 (SATF). Les notes de l'édition n'ayant jamais paru, on utilisera ici, afin de travailler sur un texte homogène, une transcription établie par nos soins du manuscrit de base de cette édition, BnF, fr. 1612; Prose 5 est en cours d'édition, cf. Anne Rochebouet, La cinquième mise en prose $d u$ Roman de Troie de Benoît de Sainte-Maure, édition partielle et commentaire, thèse pour le diplôme d'archiviste paléographe, 2005.

${ }^{5}$ Ce n'est pas le seul endroit où apparaissent des amants malheureux dans nos deux romans en prose. On y trouve également Leander, puis Brunor (Prose 1, BnF, fr. 1612, f. 81d et Prose 5, § 289), mais ceux-ci ne forment pas alors les éléments d'une liste.

6 Bernard GuENEE, Histoire et culture historique dans l'Occident médiéval, Paris, Aubier, 1980.

${ }^{7}$ Daretis Phrygii de excidio Troiae historia, recensuit Ferdinandus MEISTER, Leipzig, 1873 et Dictyis Cretensis Ephemerídos Belli Troiani libri, edidit Werner EISENHUT, Leipzig, 1973, $2^{\mathrm{e}}$ éd. (Bibiliotheca scriptorum græcorum et romanorum teubneriana). Ces deux textes sont traduits dans Récits inédits sur la Guerre de Troie, traduits et 
En dehors des insertions du récit de la guerre de Troie dans des compilations historiques, en latin comme en langue vulgaire, la matière troyenne a donné lieu à de nombreuses créations littéraires. En français, la plus ancienne et la plus diffusée est le Roman de Troie ${ }^{8}$, composé par Benoît de Sainte-Maure, un clerc tourangeau au service d'Henri II Plantagenêt, vers 1165 . On en conserve trente manuscrits complets ainsi que vingt-huit fragments. Il est copié jusqu'au $\mathrm{XIV}^{\mathrm{e}}$ siècle, notamment en Italie.

La diffusion de ce texte n'est pas seulement attestée par le nombre de manuscrits qu'on en a conservé, mais aussi par les nombreuses adaptations dont il a fait l'objet. Il a en effet été traduit en latin, sans mention de sa provenance véritable, par le juge de Messine Guido delle Colonne en 1287, sous le titre d'Historia destructionis Troiae ${ }^{9}$. Cette œuvre connaitra à son tour plusieurs traductions françaises au $\mathrm{XV}^{\mathrm{e}}$ siècle $^{10}$. Surtout, le Roman de Troie a été mis en prose à cinq reprises entre le XIII et le début du $\mathrm{XIV}^{\mathrm{e}}$ siècle. On a distingué ces mises en prose en les numérotant, leur succession numérique n'impliquant pas une succession chronologique, d'autant qu'il s'agit de textes extrêmement difficiles à dater les uns par rapport aux autres.

Prose 1 aurait été composée au XIII ${ }^{\mathrm{e}}$ siècle, peut-être en Morée franque. C'est la plus diffusée avec la cinquième prose : on en conserve dix-sept manuscrits, dont six contiennent une version remaniée du $\mathrm{XV}^{\mathrm{e}}$ siècle. Le $\mathrm{XIII}^{\mathrm{e}}$ siècle voit aussi la rédaction des deuxième, troisième et quatrième mises en prose. Prose 2 aurait été écrite dans le Nord de l'Italie, où s'est d'ailleurs limitée sa diffusion. Elle est contenue dans trois

commentés par Gérard FrY, Paris, Les Belles Lettres, « La Roue à livres », 1998.

${ }^{8}$ Léopold Constans, Le Roman de Troie par Benoît de Sainte-Maure ; publié d'après tous les manuscrits connus, Paris, F. Didot, 1904-1912. Des extraits du manuscrit $\mathrm{M}^{2}$ ont également été édités par Emmanuèle BAUMGARTNER et Françoise VIELLIARD, Le Roman de Troie. Extraits du ms Milan, Bibl. ambrosienne, D 55, Paris, Le Livre de poche, «Lettres gothiques », 1998.

9 Éd. par Nathaniel E. GRIfFIN, Cambridge (Mass.), The Mediaeval Academy of America, 1936.

${ }^{10}$ On en a jusqu'à présent distingué six, cf. Marc-René Jung, La Légende de Troie en France au Moyen Âge, analyse des versions françaises et bibliographie raisonnée des manuscrits, Basel-Tübingen, Francke, « Romanica Helvetica », 114, 1996, p. 570-601. 
manuscrits et a servi de base à un volgarizzamento que conserve un manuscrit. C'est également en Italie $^{11}$ qu'ont été réalisés le ou les plus ancien(s) témoin(s) conservé(s) de Prose 3, dont il ne nous reste plus que six fragments ${ }^{12}$, ainsi que le volgarizzamento auquel elle a donné lieu, l'Istorietta Troiana. On en conserve également un manuscrit complet du $\mathrm{XV}^{\mathrm{e}}$ siècle. Prose $4^{13}$ enfin n'est conservée que dans un seul manuscrit: racontée par Merlin et insérée dans des textes de la matière arthurienne, cette histoire de Troie serait d'origine française.

La dernière, Prose 5, aurait été composée à Naples au début du $\mathrm{XIV}^{\mathrm{e}}$ siècle : elle constitue en fait la section troyenne de la compilation d'histoire antique connue sous le nom d'Histoire ancienne jusqu'à César dans sa seconde rédaction. On en a conservé quinze manuscrits. La particularité de cette dernière prose est qu'elle n'a pas pour seul hypotexte le poème de Benoît : son rédacteur a en partie compilé Prose 1 et Prose 3 tout autant qu'il s'est servi directement du Roman en vers. Le passage qui nous intéresse est ainsi repris, et adapté, de Prose 1. Le prosateur réutilise ici une liste topique mais en l'adaptant, comme nous allons le voir, à son projet d'ensemble.

\section{De la liste d'exempla à la liste de noms : variation des amants malheureux entre Prose 1 et Prose 5}

La liste de sept amants malheureux qui fait l'objet de notre étude est une illustration de la folie engendrée par l'amour chez Achille. L'épisode prend place au cours des longues trêves accordées par les Grecs après la mort d'Hector. Achille, qui a tué le prince troyen, se rend comme tous les

11 Fabrizio Costantini, qui a consacré l'étude la plus récente à Prose 3, propose cependant de situer sa composition dans l'aire anglo-normande, "Prosa 3 di Roman de Troie : analisi sinottica fra tradizione e traduzione », Critica del testo, 7 (2004), p. 10451089.

${ }^{12}$ Ces six fragments feraient partie d'un même manuscrit, selon les travaux de Françoise VIELLIARD, «Le Roman de Troie en prose dans la version du ms. Rouen, BM O. 33, "membra disjecta" d'un manuscrit plus ancien ? », Romania, 109 (1988), p. 502-539.

${ }^{13}$ C'est la seule de ces trois proses à avoir été éditée : Le Roman de Troie en prose (Version du Cod. Bodmer 147), édité par Françoise VIELliaRD, Cologny-Genève, Fondation Bodmer, 1979. 
autres barons à la cérémonie anniversaire de la mort d'Hector. Il y aperçoit Polyxène, dernière fille de Priam, renommée pour sa très grande beauté, et il en tombe amoureux au premier regard ${ }^{14}$. Il demande alors en secret sa main, mais Hécube et Priam exigent qu'il fasse lever le siège en contrepartie. Achille convoque alors un conseil où il essaye d'imposer aux Grecs l'arrêt des hostilités et le départ des troupes, mais il est finalement débouté. Il décide de ce fait de ne plus prendre part aux combats (v. 17 489-18 442).

Le narrateur conclut cet épisode par un développement sur la folie amoureuse qui guide les actions d'Achille (v. 18 443-472, c'est nous qui soulignons) :

«C'est dévié lor fait Achillès,

Se il mesfait, qu'en puet il mais,

Quant cil li tout sen e mesure,

Qui ne garde lei ne dreiture,

Noblece, honesté ne parage?

Qui est qui vers Amors est sage?

Ço n'est il pas ne ne puet estre :

En Amors a trop grevos maistre

Trop par lit grevos leçon.

Ço parut bien a Salemon :

Mout monta poi vers lui sis sens.

De trestoz homes fait ses buens. $\gg{ }^{15}$

Benoît ne donne ici qu'une seule illustration du caractère insensé des actions provoquées par l'amour, Salomon, sous la forme d'une simple évocation. Il faut remonter de quelques centaines de vers pour trouver une courte liste de trois amants malheureux qui sert à illustrer le même comportement, mais dans la bouche d'Achille : analysant ses actions alors qu'il attend la réponse de Priam et d'Hécube à sa demande en mariage, il

\footnotetext{
${ }^{14}$ Polyxène, la plus jeune des filles de Priam et d'Hécube, ne figure pas dans l'Iliade et n'apparaît que dans les épopées postérieures. Indépendamment du récit de ses amours avec Achille et peut-être antérieurement au développement de cette légende existait dans les poètes tragiques, notamment Euripide, celle de la mort de Polyxène, sacrifiée sur la tombe du héros grec pour apaiser son âme, ou, en écho au sacrifice d'Iphigénie, pour favoriser la traversée des navires grecs (cf. Pierre GRIMAL, Dictionnaire de la mythologie grecque et romaine, Paris, PUF, 2002, $15^{\mathrm{e}}$ éd., p. 388-389).

${ }^{15}$ Éd. Constans, v. 18 443-454.
} 
revient sur la toute puissance d'Amour contre laquelle il ne peut lutter, et cite à l'appui l'exemple de Fortis Sansons, Daviz et Salemons (v. 18 044$\left.48^{16}\right)$.

La première mise en prose choisit de développer ces considérations morales: si elle conserve l'énumération des trois figures bibliques que convoque Achille dans son monologue ${ }^{17}$, elle amplifie surtout considérablement les réflexions du narrateur qui viennent clore l'épisode. Mais ce n'est pas la même facette du comportement d'Achille qui fait l'objet de l'analyse du narrateur : le prosateur s'étonne avant tout que le héros grec n'ait pas caché ses véritables intentions, échouant ainsi à obtenir l'arrêt des hostilités qu'il désirait. Cela finalement ne doit pas surprendre, car cette attitude est typique de ceux gouvernés par l'amour. Après d'assez longues remarques morales, le narrateur illustre son propos par sept exemples, tirés principalement de l'autorité par excellence, la Bible, avant d'élargir «as anciens estoires » (c'est nous qui soulignons) :

«Et en tel rage et en tel folie entra Achillés por le regart de Polexenaim, si come vos avés oï, et se mist si dou tout en pooir d'amors, que il en laissa et oblia tout son sens et sa raisnable matiere. Ce meismes est avenus plesours fois a maint saige dou Viel Testament, qui laissoient ce que lor ordene enseignoit et siveoient lor folle entention. Et ce [76c] fit tout le premier Adan, qui, par la science dou dyable et de Evain sa compaigne, trespassa le comandement Nostre Seignour. Le roi David fist tuër son seneschal por la covoitise que il ot de sa feme. Salemon li sages, par l'enortement de sa feme, laissa son sovrain Dieu a aourer et ahoura les ydeles. Sanson le fort descouvri a sa feme que il avoit la force en ses cheviaus, dont elle le fist puis honir dou cors. Le duc Oliferne, por la covoitise de Judith, reçuit mort par sa main, ja soit il chose que ce fu par la volenté Nostre Saignour, qui le vost por delivrer son pueple, mais li dus n'i avoit nulle entension se mauvaise non. Merlin, qui tant fu sages, en fu enfouis tous vis par une a qui il n'ot onques forfait. Virgilles li sages en fu escharnis et gabés laidement, selonc ce que l'en trouve as anciens estoires. Et

16 «Qui est qui contre amor est sage ?/ Ço ne fu pas Fortis Sanson,/ Li reis Daviz ne Salemon,/ Cil qui de sen fu soverains/ Sor toz autres homes humains. »

${ }^{17}$ «Fu il onques nul sages contre Amor? Ce ne fu pas le roi David, ne le sovrain Salemon. Li fors Sansons meismes ne li pot onques contrester a toute sa force » $(\mathrm{BnF}$, fr. 1612, fol. 72a). 
maint [76d] autre qui ont lor cuer en amor mis qui sont esté deceü et honis au Dieu et au siecle : dont je ne me merveil pas se Achiellés perdi la droite voie et fu si chaitif a son commencement, quar il pensoit bien que ce qui plaisoit a lui deüst plaire a tous les autres, si l'avoit Amours auveuglé. Et poés savoir que il ne fu pas le premier a cui Amor tolt sens et mesure et raison. Et ensi avient a Achillés, quar a la fin le mena tant Amors a sa volenté que il s'enivra en sa fole outrequidance, que il a la mort reçoivre en vint, si come vos porés entendre ici aprés. $»^{18}$

De la seule évocation de Salomon, on est passé à une mise en série de sept figures exemplaires, accompagnées d'un bref récit de l'événement qui justifie leur inscription dans cette séquence. Achille, en dernier lieu, y est également bien intégré (« Et poés savoir que il ne fu pas le premier a cui Amor tolt sens et mesure et raison »).

Ce développement spécifique au premier prosateur est à rapprocher, plutôt qu'à la thématique des Hommes illustres, à la pratique médiévale de l'exemplum $^{19}$. On doit également le replacer dans le projet général de l'œuvre : le rédacteur de Prose 1 se veut avant tout historien, et donc, selon la conception médiévale de l'histoire, moralisateur ${ }^{20}$ : son but est d'instruire par les exemples passés. Il use ainsi de l'attirail habituel des moralistes pour extraire la signification morale du récit qu'il relate à travers ce qu'il appelle lui-même des « essamples $»^{21}$, qui sont à la fois des exempla et les leçons qu'il faut en tirer.

Le choix des figures vétéro-testamentaires n'est pas original: on les retrouve dans la plupart des listes de ce type ${ }^{22}$. Les deux derniers personnages méritent que l'on s'y attarde davantage. La référence à Merlin

${ }^{18} \mathrm{BnF}$, fr. 1612, f. 76b-c, qui correspond au $§ 198$ de l'édition CONSTANS-FARAL.

${ }^{19}$ Rappelons ici la définition qu'en a donnée de Jacques Le Goff : « récit bref donné comme véridique et destiné à être inséré dans un discours, en général un sermon, pour convaincre un auditoire par une leçon salutaire ».

${ }^{20}$ Françoise ViELliarD, « Du Roman de Troie à la « veraie estoire de Troie » (Prose 1 version commune) : le choix de l'Histoire », in Laurence HARF-LANCNER, Laurence Mathey-Maille et Michelle Szkilnik, Conter de Troie et d'Alexandre, Paris, Presses Sorbonne Nouvelle, 2006, p. 177-193.

${ }^{21}$ On en trouve aux fol. 10b, 75d, 98d et $112 \mathrm{~d}$.

${ }^{22}$ Claude BURIDANT signale dans son introduction à la traduction du Traité de l'amour courtois d'André le Chapelain que ce sont Urié, David et Salomon les plus fréquemment cités (Paris, Klincksieck, 1974, p. 36). 
renvoie à l'univers littéraire fictionnel ${ }^{23}$ et pourrait montrer, de la part du prosateur, une volonté de séduire son public par l'évocation d'une lecture de «distraction ». Mais Merlin est également au Moyen Âge l'auteur de prophéties, ce qui justifie son insertion au milieu des autres personnages. L'épisode mettant en scène Virgile auquel il est fait ici référence remonte à la tradition tardive latine ${ }^{24}$, où apparait, à partir du $\mathrm{XII}^{\mathrm{e}}$ siècle, un Virgile magicien ou philosophe, mais amoureux et donc tourné en dérision. Épris de Fébille, une courtisane romaine, il accepte de monter dans la tour où elle vit par une corbeille, mais elle ne le hisse pas jusqu'en haut et il reste suspendu à mi-chemin. Si l'on met à part cet épisode peu édifiant, Virgile est avant tout considéré comme un savant philosophe. Les sept figures sont donc choisies parmi des hommes sages et savants. L'accumulation des exemples fait preuve et renforce l'argument d'autorité ; elle ne semble pas relever d'une volonté de distraire ou de captiver le lecteur.

L'adaptation qu'en fait le rédacteur de Prose 5 (qui se sert ici, comme on l'a dit plus haut, de Prose 1) est très différente. Il reprend les modifications apportées par le premier prosateur: ce qui est reproché à Achille est d'avoir dévoilé d'entrée de jeu sa volonté, ruinant ainsi ses chances. Mais le cinquième prosateur réduit ensuite considérablement les remarques morales (notamment les références aux actions des «dyables» qui guident les actions des hommes) pour en venir assez rapidement à l'illustration de son propos : il cite pour cela sept figures à nouveau, mais sans donner le récit de l'épisode qui justifie leur inclusion dans sa liste (c'est nous qui soulignons) :

«En tel folie entra Achillés par le regart de Pollicena si con vous avés oï, et si se mist si du tout el pooir d'Amour que il oublia son sens. Et ce meïsmes est avenu a pluseurs sages du Viel Testament,

${ }^{23}$ Il s'agirait ici plutôt d'une référence au Merlin-Huth où Merlin est enfoui sous des pierres, qu'à la Suite Vulgate, où il est enserré dans une prison d'air.

${ }^{24}$ Cet épisode est attribué à Hippocrate dans l'Estoire del Saint Graal de Robert de Boron, à Antipater dans les Proverbia quae dicuntur super natura feminarum et à Guiraut de Calanso dans son Fadet Joglar Libro de buen amor. On le retrouve, mettant en scène Virgile chez Jean d'Outremeuse, mais aussi dans des recueils d'exempla, comme les sages de l'Ancien Testament cités plus haut (cf. Thesaurus exemplorum Medii Aevi, consultable en ligne http://gahom.ehess.fr). 
et ce fist nostre premier pere Adan, li rois David et li sages Salemon, Sanson li fors et li dux Olifernes, Virgile et Aristotes. Tous ceuls qui furent la fleur du monde de sens et de force furent par feme souspris et engenné, pour quoi je ne me merveil pas se Achillés fu hors de la droite voie et se Amour le mena si par sa force que il en perdi ci endroit son sens propre et naturel, et a la fin en perdi il la vie, si con vous porréz oïr. $»^{25}$

On aboutit ainsi à une liste de noms qui, par la suppression des récits qui les accompagnaient, sont redevenus de simples évocations d'un renom négatif. Par là, dans la version qu'en donne Prose 5 qui, rappellonsle, aurait été composée, au moins dans la version qui nous est parvenue, en Italie, cette liste me paraît être un timide reflet de la thématique des Hommes illustres qui se développe au même moment en Italie.

\section{Un timide reflet de la thématique des Hommes illustres?}

On est en effet passé, entre les deux proses, d'une série d'exempla à une simple liste de noms. Or, comme on l'a rappelé plus haut, avant le XVI siècle où se multiplient en France les recueils de vies d'Hommes illustres, c'est la liste, parallèlement au recueil de biographies et à divers catalogues qui existent déjà, qui semble servir le plus de mise en forme et de lieu d'expression à la thématique des Hommes illustres, et ce à partir du XIV siècle. Jacqueline Cerquiglini-Toulet a ainsi montré « qu'il fleurit [...] dans les textes du XIV ${ }^{\mathrm{e}}$ siècle des listes de noms qui sont des cristallisations de renom ${ }^{26}$.

L'accumulation des courts récits dans Prose 1 donnait l'impression d'un enchaînement fragmenté d'épisodes narratifs ; toute narration est à l'inverse supprimée dans Prose 5 où ces noms deviennent des évocations et non des illustrations. Ils se suffisent à eux seuls car ils sont des incarnations de renom. Le prosateur dresse ainsi en quelque sorte un panthéon d'amants malheureux, dont le caractère illustre ne fait aucun doute : il s'agit de « tous ceuls qui furent la fleur du monde de sens et de force ». Il constitue bien

\footnotetext{
${ }^{25}$ Éd. Anne Rochebouet, § 280.

${ }^{26}$ Jacqueline CERquiglini-Toulet, « $\mathrm{A}$ la recherche des pères : la liste des auteurs illustres à la fin du Moyen Âge », MLN 116 (2001), p. 636.
} 
une lignée de figures contre-exemplaires, dont il faut tout faire, si cela est possible, pour se détacher.

Le remplacement de Merlin au profit d'Aristote nous parait aller dans le même sens. L'anecdote à laquelle il est fait référence est à rapprocher de celle de Virgile, à laquelle elle est d'ailleurs souvent associée. Elle apparaît en Occident avec Le Lai d'Aristote, composé au $\mathrm{XIII}^{\mathrm{e}}$ siècle par Henri de Valenciennes, qui raconte les mésaventures d'Aristote et de la maîtresse d'Alexandre, Phyllis. L'épisode a connu un grand succès ${ }^{27}$, notamment iconographique. La référence à ces deux épisodes est bien sûr à rapprocher de la littérature anti-féministe, plutôt cléricale, mais tout aussi abondante en latin qu'en langue vulgaire ${ }^{28}$. Mais on peut aussi y voir le remplacement de Merlin, qui reste avant tout un personnage de fiction, par une figure dont l'historicité ne peut être mise en doute : Prose 5 présente une série de personnages véritablement réels, dont l'inscription historique en fait des modèles (ici à rebours) potentiels.

Les variations que connaît notre liste d'amants malheureux dans ces deux textes de la matière troyenne, la première et la cinquième mises en prose, nous semblent refléter l'apparition de la thématique des Hommes illustres. D'un enchaînement d'exempla à vocation purement moralisante, on passe en effet à une mise en série de noms qui se suffisent à eux-mêmes. «Fine fleur» de l'humanité, et pourtant victimes d'Amour et des comportements insensés qu'il provoque, ils constituent une lignée illustre à la suite de laquelle le lecteur est exhorté à ne pas s'inscrire.

\footnotetext{
${ }^{27}$ L'épisode a aussi été utilisé comme exemplum, par exemple par Jacques de Vitry (cf. base de données THEMA, citée supra).

${ }^{28}$ Claude Buridant (éd.), ANDre le Chapelain, Traité de l'amour courtois, op. cit., p. 35-37.
} 